\title{
A new emerging oral infection: Raoultella planticola in a boy with haematological malignancy
}

\author{
E. Bardellini ${ }^{1}$ F. Amadori ${ }^{1}$ - R. F. Schumacher ${ }^{2}$ I. Foresti $^{3}$ - A. Majorana ${ }^{1}$
}

Received: 23 May 2016/ Accepted: 18 February 2017

(C) European Academy of Paediatric Dentistry 2017

\begin{abstract}
Background Oral mucositis is a common complication in pediatric cancer patients, affecting up to $80 \%$ of children. Due to neutropenia and disruption of the mucosal barrier, chemotherapy-induced oral mucositis is often complicated by super-infections.

Case report A 16-years old male with stage 3 Burkitt's lymphoma developed chemotherapy induced oral mucositis grade 3 (according to WHO scale). Ulcers were quickly growing (reaching a maximum diameter of $3 \mathrm{~cm}$ ) and became greyish in colour, resulting in dysphagia and pain. A swab of the lesions was taken and microbiological tests were performed. The sample grew for Raoultella planticola, an encapsulated Gram-negative bacterium whose full pathogenic potential still needs to be defined. Treatment: The patient received antibiotic combination therapy with Amikacin and Ceftazidime for 8 days. Complete healing of the lesions and resolution of the symptoms were reached and he completed his antineoplastic therapy without further complications. Follow-up: Twelve months after the infection, he is alive and well, with no oral complaints.

Conclusion This is the first report of a Raoultella planticola infection in a patient with chemotherapy induced oral mucositis. This type of infection must be added to the list
\end{abstract}

E. Bardellini

elena.bardellini@unibs.it

1 Department Oral Medicine and Paediatric Dentistry, Dental Clinic, University of Brescia, p.le Spedali Civili n.1, 25133 Brescia, Italy

2 Paediatric Haematology-Oncology Unit, Spedali Civili di Brescia, Brescia, Italy

3 Microbiology Department, Spedali Civili di Brescia, Brescia, Italy of organisms to be considered when caring for these patients.

Keywords Oral ulcer · Infection · Anti-neoplastic agents

\section{Background}

While early diagnosis and advances in cancer therapy for children continue to improve survival rates, oral complications remain a significant cause of morbidity and potential mortality. Oral mucositis (OM) is a common complication in cancer patients receiving chemotherapy with an incidence in the paediatric cancer population of 50-80\% (Bardellini et al. 2013). OM initially presents as erythema of the oral mucosa, which then often progresses to erosion and ulceration; it can be very painful and it can significantly affect nutritional intake, mouth care, and overall quality of life, not to forget lengthening of hospital stay and associated costs. In patients receiving chemotherapy for solid tumours or lymphoma, the rate of infection during cycles with mucositis is more than twice that during cycles without mucositis and directly proportional to the severity of mucositis (Elting et al. 2003). Infection-related deaths are also more common during cycles with both oral and gastro-intestinal mucositis. In addition, the average duration of hospitalisation is significantly longer during chemotherapy cycles with mucositis. A reduction in the next dose of chemotherapy is twice as common after cycles with mucositis than after cycles without mucositis (Elting et al. 2003). Thus, mucositis and related infections may lead to dose-limiting toxicity of chemotherapy with fatal effects on patient survival.

Raoultella planticola (RP) — formerly known as Klebsiella planticola-is a Gram-negative, non-motile, 
facultative aerobic and lactose fermenting bacillus that is primarily considered an environmental bacteria, belonging to the Enterobacteriaceae family (Freney et al. 1984). Raoultella planticola is mostly an aquatic, botanical and soil organism and, usually, it is not considered a cause of invasive infections in humans. Raoultella species produce histidine decarboxylase and have been implicated in scombroid (histamine) fish poisoning (Lam and Salit 2014). Long considered almost harmless to humans, this environmental organism only recently has been reported as the cause of several infections. The first case-report appeared in 1984; however the pathogen responsible for the infection was thought to be a Klebsiella species (Freney et al. 1984). Only in 2001 was this pathogen reclassified as belonging to a new different genus (Drancourt et al. 2001). Several studies report that $9-18 \%$ of humans are colonised by this bacterium (Freney et al. 1984; Podschun et al. 1998; Westbrook et al. 2000). It has to be noted that RP shares strong similarities with $K$. pneumoniae, in terms of pathogenicity, virulence and drug-resistance mechanisms (Murray et al. 2007). Raoultella planticola is found to have similar pathogenetic features as Klebsiella spp. which are associated with severe infections in fragile patients, as: bacteraemias, pneumonias, and urinary tract infections (Olson et al. 2013; Cho et al. 2016).

Common virulence factors between Klebsiella spp. and RP has been investigated finding that both, Klebsiella pneumoniae and RP, have type 1 fimbriae and mannosesensitive haemagglutination; furthermore Klebsiella spp. have the ability to resist the bactericidal effects of human serum, a feature that is shared by RP (Mertens et al. 2010).

This paper describes the case of a haemato-oncologic patient, suffering from chemotherapy induced oral mucositis, who developed a RP infection in the oropharynx. To our knowledge no previous case, in terms of pathogen and localisation, has been reported.

\section{Case report}

A 16-years-old boy was hospitalised with a diagnosis of stage 3 Burkitt's lymphoma, treated following the AIEOP LNH97 therapeutic protocol (risk group 2), which includes twice weekly Cotrimoxazole prophylaxis. According to the Multinational Association of Supportive Care in Cancer and International Society for Oral Oncology (MASCC/ ISOO) guidelines on dental management of paediatric patients receiving chemotherapy, the boy underwent a dental assessment before the chemotherapy. No caries or periodontal lesions were detected and the oral hygiene grade was good. During the second cycle A (an intense 5 days combination of Vincristine, Methotrexate, Ifosfamide, Cytarabine and Etoposide) the patient developed a chemotherapy-induced oral mucositis grade 3 , according to WHO scale (Sonis et al. 2004). Oral manifestations included atrophic lesions of the cheeks, erosions of the palate and of the lips, ulcers on both palatine pillars; associated symptoms were pain and extreme difficulty in chewing and swallowing food (Fig. 1). To exclude a concomitant bacterial infection, a swab of the lesions was taken. After 2 days the sample grew RP. An antibiogram was performed (ST-N204 Vitek-II card, based on the EUCAST guideline), resulting in a completely sensitive bacterium, with good susceptibility to all the tested antibiotics, including Cotrimoxazole (MIC $\leq 20 \mu \mathrm{g} / \mathrm{mL}$ ). In the meantime the ulcers had grown in size (almost $3 \mathrm{~cm}$ after 5 days) and turned grey in colour, leading to increased dysphagia and pain (severity 8/10). The patient also experienced a febrile neutropaenia $\left(0.05 \times 10^{9} \mathrm{WBC} / \mathrm{L}\right)$. $\mathrm{He}$ had a C-reactive protein of $160 \mathrm{mg} / \mathrm{L}$ and a procalcitonin of $0.6 \mu \mathrm{g} / \mathrm{L}$.

\section{Treatment}

Following assessment of the antibiogram, a combination therapy with intravenous Amikacin and Ceftazidime was commenced. The patient also received G-CSF and his fever resolved within $72 \mathrm{~h}$. The boy underwent a complete biochemical assessment with repeated blood and urine cultures, viral PCR and multiple swabs without any other positive finding. Complete healing of the mucosal lesions and resolution of the symptoms were reached after 8 days of antibiotics (Fig. 2).

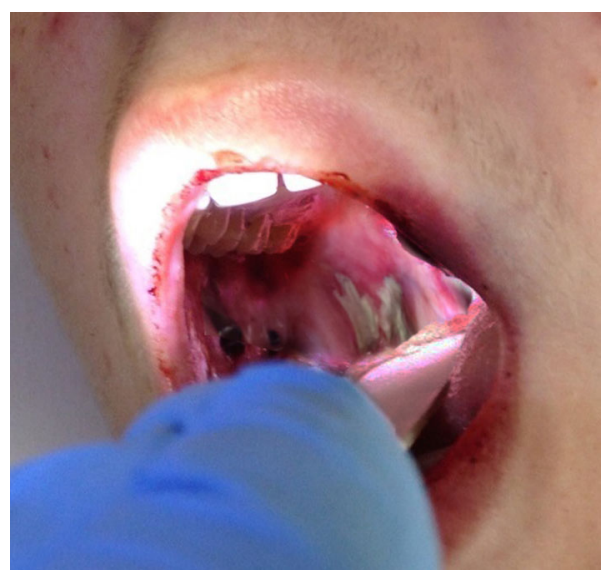

Fig. 1 Sixteen-years-old male with chemotherapy-induced oral mucositis grade 3 , showing atrophic lesions of the cheeks, erosions of the palate and of the lips, with ulcers on both palatine pillars 


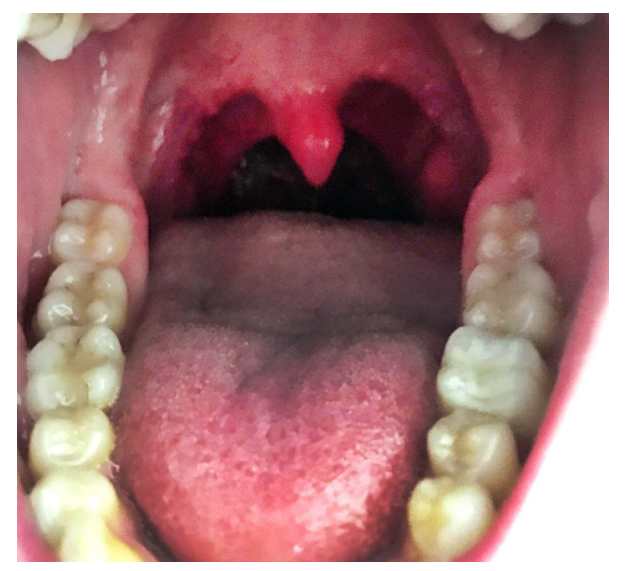

Fig. 2 Complete healing of the mucosal lesions and resolution of the symptoms after 8 days

\section{Follow-up}

The boy was discharged free of symptoms, with normal blood values and continued his chemotherapy as scheduled without delay. Now, 12 months after the infection, he is alive and well, with no oral complains.

\section{Discussion}

The development of new chemotherapeutic and biological agents has resulted in a substantial decrease in cancer related death rates. Unfortunately, these treatment options are often associated with profound immunosuppression, mucosal damage and thus carry an increased risk of infection. Neutropaenia-not only quantitative but also qualitative-remains the most common adverse event of anti-neoplastic chemotherapy (Nesher and Rolston 2014). These defects include significant reduction in phagocytosis, decreased bactericidal and fungicidal activity, decreased production of superoxide anions, and defects in granulocyte locomotion. Infectious events can negatively impact survival directly and indirectly by causing dose reduction or delays in anti-neoplastic therapy. Under strict hygiene measures, most microbiologically documented infections arise from the patient's endogenous micro-flora, with only a small proportion being acquired from exogenous sources or as a result of environmental exposure. Patients with severe oral mucositis are likely to have infections caused by viridans group Streptococci (VGS) and Stomatococcus mucilaginosus but coagulase-negative Staphylococci, Klebsiella pneumoniae, Pseudomonas aeruginosa, and Escherichia coli are also often encountered beside microorganisms of the normal oral flora (Nesher and Rolston 2014). Several recent epidemiologic surveys in adult and paediatric patients from various parts of the globe have documented the predominance of Gram-positive organisms over Gram-negative bacilli as causes of microbiologically documented infections in neutropaenic patients (Nesher and Rolston 2014). Some of the reasons for this predominance include the almost universal use of central venous catheters in such patients, the use of antimicrobial prophylaxis directed primarily against enteric Gram-negative bacilli, and last but not least the frequent use of chemotherapy that produces significant oral mucositis (Nesher and Rolston 2014).

Raoultella planticola is an emerging pathogen: in a review conducted by Ershadi et al. (2014), a total of 17 cases of severe infection (dated from 1984 to 2014) were reported, i.e. six patients with bacteraemia, four patients with infections of the gastrointestinal tract, three patients with skin and soft tissues infections, three patients with pneumoniae and one patient with cystitis. Nine infections were confirmed to be nosocomial, six of which involved immunocompromised patients. Three patients who developed bacteraemia died, due to multi-drug resistance strains. Various authors suggest at least four possible scenarios that could make a patient develop an infection with this pathogen: (a) inoculation of a significant load of bacteria in a immunologically and/or anatomically injured site, due to trauma in a contaminated environment (community acquired; O'Connell et al. 2010; Kim et al. 2012); (b) introduction of the pathogen through invasive hospital procedures (nosocomial infection; Freney et al. 1984; Kim et al. 2012; (c) activation of dormant colonies in immunocompromised patients (Alves et al. 2007; Yokota et al. 2012) (d) enteric fever and bacteraemia in immunocompetent individuals (fish poisoning; Puerta-Fernandez et al. 2013). Extended post-hoc anamnesis did not reveal any recent fish intake (though he had close contact with farm animals) or particular trauma prior to chemotherapy, which makes reactivation of dormant colonies the most probable mechanism in this patient.

\section{Conclusions}

One of the frequently overlooked complications of chemotherapy is oral mucositis. This condition can range from mild to severe and represents a common cause of chemotherapy dose reduction and treatment delays as well as infections. Chemotherapy-related oral infections, which account for $25-50 \%$ of the total infections, contribute significantly to the morbidity and mortality in these patients. This case report highlights the need to now add Raoultella planticola to the list of bacteria encountered in the course of oral mucositis in patients undergoing chemotherapy. 


\section{Compliance with ethical standards}

Conflict of interest The Authors declare that they have no conflict of interest.

Ethical standard All procedures performed in this study were in accordance with the ethical standards of the Declaration of Helsinki.

Informed consent Informed consent from the patient's parents for the publication of the case was taken.

\section{References}

Alves MS, Riley LW, Moreira BM. A case of severe pancreatitis complicated by Raoultella planticola infection. J Med Microbiol. 2007;56:696-8.

Bardellini E, Schumacher F, Conti G, et al. Risk factors for oral mucositis in children receiving hematopoietic cell transplantation for primary immunodeficiences: a retrospective study. Pediatr Transpl. 2013;17:492-7.

Cho YJ, Jung EJ, Seong JS, et al. A case of pneumonia caused by Raoultella planticola. Tuberc Respir Dis (Seoul). 2016;79:42-5.

Drancourt M, Bollet C, Carta A, Rousselier P. Phylogenetic analyses of Klebsiella species delineate Klebsiella and Raoultella gen. nov., with description of Raoultella ornithinolytica comb. nov., Raoultella terrigena comb. nov. and Raoultella planticola comb. nov. Int J Syst Evol Microbiol. 2001;51:925-32.

Elting LS, Cooksley C, Chambers M, et al. The burdens of cancer therapy. Clinical and economic outcomes of chemotherapyinduced mucositis. Cancer. 2003;98(7):1531-9.

Ershadi E, Weiss E, Verduzco D, Chia D, Sadigh M. Emerging pathogen: a case and review of Raoultella planticola. Infection. 2014;42(6):1043-6.

Freney J, Fleurette J, Gruer LD, et al. Klebsiella trevisanii colonisation and septicaemia. Lancet. 1984;1:909.

Kim SH, Roh KH, Yoon YK, et al. Necrotizing fasciitis involving the chest and abdominal wall caused by Raoultella planticola. BMC Infect Dis. 2012;12:59.
Lam PW, Salit IE. Raoultella planticola bacteremia following consumption of seafood. Can J Infect Dis Med Microbiol. 2014;25(4):e83-4.

Mertens K, Mns K, Loennies S, et al. Antiserum against Raoultella terrigena ATCC 33257 identifies a large number of Raoultella and Klebsiella clinical isolates as serotype O12. Innate Immun. 2010;16(6):366-80.

Murray PR, Baron EJ, Jorgensen JH, Pfaller MA, Yolken RH. Manual of clinical microbiology. 8th ed. Washington DC: ASM Press; 2007.

Nesher L, Rolston KV. The current spectrum of infection in cancer patients with chemotherapy related neutropenia. Infection. 2014;42(1):5-13.

O'Connell K, Kelly J, Niriain U. A rare case of soft-tissue infection caused by Raoultella planticola. Case Rep Med. 2010;. doi:10. 1155/2010/134086 (epub 2010 Aug 1).

Olson DS Jr, Asare K, Lyons M, Hofinger DM. A novel case of Raoultella planticola urinary tract infection. Infection. 2013;41(1):259-61.

Podschun R, Acktun H, Okpara J, et al. Isolation of Klebsiella planticola from newborns in a neonatal ward. J Clin Microbiol. 1998;36:2331-2.

Puerta-Fernandez S, Miralles-Linares F, Sanchez-Simonet MV, Bernal-Lopez MR, Gomez-Huelgas R. Raoultella planticola bacteraemia secondary to gastroenteritis. Clin Microbiol Infect. 2013;19:E236-7. doi:10.1111/1469-0691.12102

Sonis ST, Elting LS, Keefe D, et al. Perspectives on cancer therapyinduced mucosal injury: pathogenesis, measurement, epidemiology, and consequences for patients. Cancer. 2004;100:1995-2025.

Westbrook GL, O'Hara CM, Roman SB, Miller JM. Incidence and identification of Klebsiella planticola in clinical isolates with emphasis on newborns. J Clin Microbiol. 2000;38:1495-7.

Yokota K, Gomi H, Miura Y, Sugano K, Morisawa Y. Cholangitis with septic shock caused by Raoultella planticola. J Med Microbiol. 2012;61:446-61. 